\title{
Using Centromere Mediated Genome Elimination to Elucidate the Functional Redundancy of Candidate Telomere Binding Proteins in Arabidopsis thaliana
}

\author{
Nick Fulcher ${ }^{1}$ and Karel Riha ${ }^{2 *}$ \\ ${ }^{1}$ Gregor Mendel Institute, Austrian Academy of Sciences, Vienna, Austria, ${ }^{2}$ Central European Institute of Technology, \\ Masaryk University, Brno, Czech Republic
}

Proteins that bind to telomeric DNA form the key structural and functional constituents of telomeres. While telomere binding proteins have been described in the majority of organisms, their identity in plants remains unknown. Several protein families containing a telomere binding motif known as the telobox have been previously described in Arabidopsis thaliana. Nonetheless, functional evidence for their involvement at telomeres has not been obtained, likely due to functional redundancy. Here we performed genetic

OPEN ACCESS

Edited by:

Arthur J. Lustig,

Tulane University, USA

Reviewed by:

Antonella Sgura,

Roma Tre University, Italy

Michael McEachern,

University of Georgia, USA

*Correspondence:

Karel Riha

karel.riha@ceitec.muni.cz

Specialty section:

This article was submitted to

Genetics of Aging,

a section of the journal

Frontiers in Genetics

Received: 06 September 2015

Accepted: 29 November 2015

Published: 05 January 2016

Citation:

Fulcher N and Riha K (2016) Using

Centromere Mediated Genome

Elimination to Elucidate the Functional

Redundancy of Candidate Telomere

Binding Proteins in Arabidopsis

thaliana. Front. Genet. 6:349.

doi: 10.3389/fgene.2015.00349 analysis on the TRF-like family consisting of six proteins (TRB1, TRP1, TRFL1, TRFL2, TRFL4, and TRF9) which have previously shown to bind telomeric DNA in vitro. We used haploid genetics to create multiple knock-out plants deficient for all six proteins of this gene family. These plants did not exhibit changes in telomere length, or phenotypes associated with telomere dysfunction. This data demonstrates that this telobox protein family is not involved in telomere maintenance in Arabidopsis. Phylogenetic analysis in major plant lineages revealed early diversification of telobox proteins families indicating that telomere function may be associated with other telobox proteins.

Keywords: telomeres, centromere, haploid, telobox, protein family

\section{INTRODUCTION}

Telomeres represent the nucleoprotein complexes that cap natural chromosome ends and function in the suppression of DNA damage signaling and control of cellular senescence. The classical telomere structure comprises tandem arrays of TTAGG-like sequences which contain G-rich $3^{\prime}$ overhangs at their termini. TRF1 and TRF2 represent the core duplex binding proteins of the mammalian telomere capping complex known as shelterin (de Lange, 2005); TRF1 is thought to be a regulator of telomere length (van Steensel and de Lange, 1997) and TRF2 has been shown to play a central role in protecting chromosome ends from end to end fusions and recombination (van Steensel et al., 1998; Wang et al., 2004). In contrast to the situation in a number of eukaryotic organisms which have extensively characterized chromosome-end capping protein complexes, the plant telomere binding components remain elusive (Watson and Riha, 2010). A hallmark of telomere binding proteins includes the presence of a single Myb domain containing the telobox, a motif that provides specificity to the telomeric sequence (Bilaud et al., 1996). Telobox containing proteins (TRF-like, TRFL) are present in genomes of all major groups of eukaryotes and they have 
been considered the prime suspects for bona fide telomere binding proteins in plants. Indeed, functional analysis of TRFL proteins in rice and tobacco has indicated their involvement in telomere length homeostasis (Yang et al., 2004; Hong et al., 2007).

TRFL proteins have been extensively studied in Arabidopsis. The Arabidopsis thaliana genome encodes at least 15 proteins containing a single Myb domain with the telobox that are divided into three families (Zellinger and Riha, 2007). The Smh/TRB family consists of three proteins harboring a histone H1-like motif involved in multimerization, and the Myb domain at the N-terminus (Marian et al., 2003; Kuchar and Fajkus, 2004; Mozgova et al., 2008). The second family includes six proteins (TRFL3, 5, 6, 7, 8, 10; TRFL Group II) that are unable to bind telomeric DNA in vitro, and are also unable to form homo- and heterodimers, despite possessing the C-terminal Myb-telobox domain (Karamysheva et al., 2004). The third family also consists of six proteins with the C-terminal Myb domain (TBP1, TRP1, TRFL1, TRFL2, TRFL4 and TRFL9; TRFL Group I), but these proteins homo- and heterodimerize and can efficiently bind to telomeric DNA in vitro (Karamysheva et al., 2004). A key feature of this family is a $\sim 30$ amino acid extension of the Myb-telobox domain that is likely responsible for specific binding to plant telomeric DNA. Structural studies of related tobacco and rice TRFL proteins determined that their binding to telomeric DNA occurs in a similar fashion as for human TRF1 (Ko et al., 2008, 2009). Thus, members of the TRFL Group I family have long been considered to act as putative telomere binding proteins in Arabidopsis. Nevertheless, plants containing single knockouts within members of this gene family have not shown drastic telomeric phenotypes (Karamysheva et al., 2004). The lack of severe telomere related phenotypes similar to mammalian TRF2 knock-outs suggested a functional redundancy among these proteins in Arabidopsis.

Reverse genetics based approaches have been used over many studies in Arabidopsis to target functional redundancy amongst gene families. Construction of lines with multiple T-DNA insertions in desired genes can, however, be time consuming requiring extensive genotyping of large populations of recombinant plants. Methods to improve the production of such mutant lines would be greatly beneficial to elucidate functional redundancy within gene families. Centromere mediated genome elimination has proven to be a powerful tool in Arabidopsis genetics allowing generation of haploid plants, rapid production of recombinant inbred lines, and reverse breeding approaches (Ravi and Chan, 2010; Seymour et al., 2012; Wijnker et al., 2012; Ravi et al., 2014). Crossing fertile male plants to the female cenh3/GFP-tailswap haploid inducer allows for the segregation of haploid plants containing genomes from the male parent. This technology also has the potential to easily generate multiple homozygous mutant combinations when crossing plants segregating for numerous T-DNA insertions to the haploid inducer (Ravi et al., 2014). In this case, haploid plants with interesting combinations can be analyzed directly for phenotypic defects, or diploids can also be recovered in the next generation due to spontaneous diploidization. This process would greatly reduce the genotyping workload that is normally associated with the generation of quadruple or sextuple mutants by selfing alone.

In this study we tackled the functional redundancy thought to occur in Arabidopsis TRFL Group I family by using production of haploid plants via centromere mediated genome elimination. We have demonstrated that this method substantially facilitates generation of multiple quadruple, quintuple and sextuple mutants. Surprisingly, results show that multiple mutants do not display drastic telomeric length defects as shown for the mutants in other genes known to act at telomeres. This demonstrates that, at least in Arabidopsis, the TRFL protein family harboring the Myb extension does not contribute to telomere protection and/or maintenance. Furthermore, this study shows another use for centromere mediated genome elimination in the production of lines containing multiple mutations.

\section{MATERIALS AND METHODS}

\section{Plant Lines}

All T-DNA insertions used are shown in Supplementary Table S1 and Supplementary Figure S1. The tbp1-1 mutant was obtained from the Institut National de la Recherche Agronomique Versailles (INRAV) collection and other alleles were from the European Arabidopsis stock centre (NASC). Plants were grown at $22^{\circ} \mathrm{C}$ in $16 \mathrm{~h}$ light $/ 8 \mathrm{~h}$ dark cycles.

\section{Centromere Mediated Genome Elimination}

The cenh3/GFP-tailswap haploid inducer line was described previously by (Ravi and Chan, 2010). Homozygous cenh3 mutant plants were confirmed by PCR genotyping using derived Cleaved Amplified Polymorphic Sequence (dCAPS) oligos (5' GGTGCGATTTCTCCAGCA GTAAAAATC-3' and 5'-CTGAG AAGATGAAGCACCGGCGATAT-3'). Resulting PCR products were digested with EcoRV, cleaved wild type (WT) alleles produced 191 and $24 \mathrm{bp}$ fragments.

Haploid inducer cenh3/GFP-tailswap lines are mostly male sterile, but can be crossed as female. Heterozygous quadruple or sextuple mutants were crossed to cenh3/GFP-tailswap lines to produce haploid offspring that were homozygous for a combination of insertions derived from the male parent. Only plants that displayed the haploid phenotype as described by Ravi and Chan (2010) were selected for further analysis. These haploids were then subject to PCR genotyping using oligos shown in Supplementary Table S1. Diploid seeds can then be recovered from haploid plants due to spontaneous diploidization which allowed analysis of subsequent generations.

\section{DNA Extraction and Telomere Analysis}

One to two leaves were homogenized in $500 \mu$ l Extraction buffer (0.2 M Tris pH9, 0.3 M LiCl, $25 \mathrm{mM}$ EDTA, and 1\% SDS) tubes were centrifuged for $10 \mathrm{~min}$ at $4000 \mathrm{rpm}(\mathrm{rcf} 1756 \mathrm{~g})$ and $350 \mu \mathrm{l}$ was transferred to $350 \mu \mathrm{l}$ isopropanol. Tubes were inverted to mix and centrifuged for $20 \mathrm{~min}$ at $4000 \mathrm{rpm}$. Supernatant was poured 
away and the pellet was washed with $70 \%$ Ethanol. The remaining

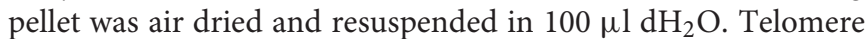
length was determined by terminal restriction fragment analysis, and statistical analysis of telomeric smears was performed using the TeloTool software (Gohring et al., 2014; Fulcher et al., 2015). Integrity of blunt ended telomeres was determined as previously described (Kazda et al., 2012).

\section{Phylogenetic Analysis}

Sequences of telobox containing proteins were obtained from indicated plant genomes by using http://www.phytozome.net, protein BLAST searches with the A. thaliana TRFL6 protein sequence as a query. Proteins were aligned by the ClustalW method and phylogenetic trees were constructed by Neighbor Joining method using CLC Main Workbench software (Qiagen).

\section{RESULTS}

\section{Knockouts of TBP1 and TRFL9 Showed No Changes in Telomere Length and Blunt End Distribution}

Phylogenetic analysis indicated that $A$. thaliana Group I TRFL proteins result from relatively recent duplication events in Brasicaceae (Figure 1). Therefore, some paralogs may still retain overlapping functions. To begin elucidating the role of TRFL proteins at telomeres, we first examined the published allele of tbp1-1 which has been reported to show telomere

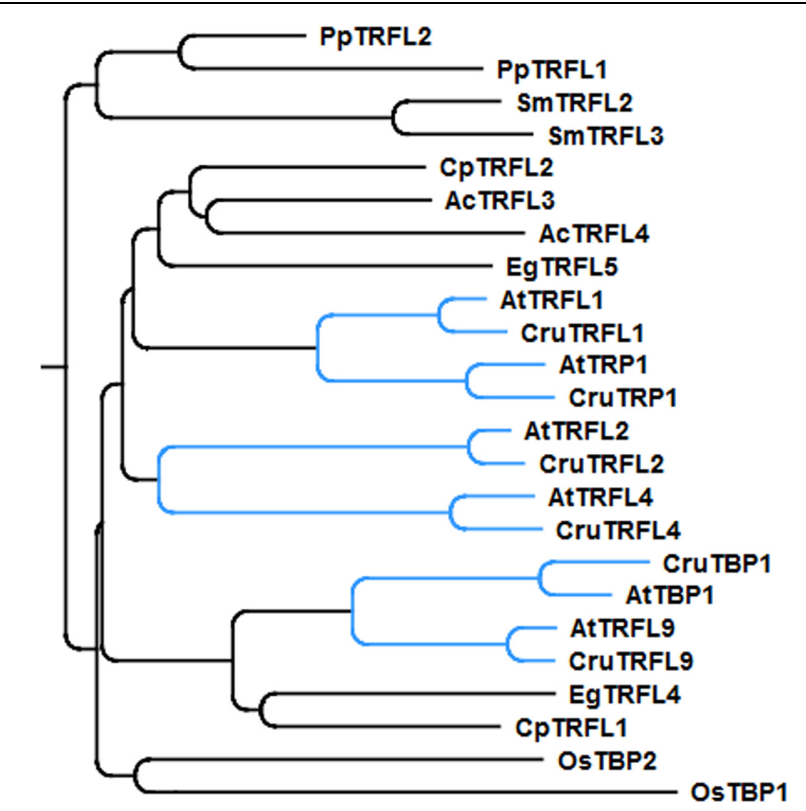

FIGURE 1 | Phylogenetic tree of the Group I TRFLs (Ac, Aquilegia coerulea; At, Arabidopsis thaliana; $\mathrm{Cp}$, Carica papaya; $\mathrm{Cr}$, Capsella rubella; Eg, Eucalyptus grandis; Os, Oryza sativa; Pp, Physcomitrella patens; Sm, Selaginella moellendorffii). Clade Brasicaceae is indicated in blue. elongation (Hwang and Cho, 2007). Within the TRFL family, TBP1 contains a closely related family member, TRFL9, which displays a high level of sequence conservation (Figure 1). We reasoned that double knockouts could exacerbate $t b p 1-1$ telomere phenotypes. Heterozygous plants containing the published tbp11 allele (FLAG_072C05) were crossed to plants heterozygous for the trfl9 (GK-036D11) mutation. Double heterozygous F1 plants were then selfed and First generation WT, double, and single mutants were segregated. DNA from five pooled plants was extracted from second and third generation of double mutants of the same lineage and subject to TRF analysis (Figure 2). To extract data from TRF blots, we used the recently published software TeloTool to measure telomere length and create graphs to better illustrate mean and range of telomeric smears (Gohring et al., 2014). No difference in telomere length was observed in second and third generation tbp 1-1 mutants compared to WT plants segregated from the same cross (Figures 2A,B). Double tbp1 trfl9 mutants also did not appear to shown any great change in telomere length over three generations. Previous studies have shown that telomere lengthening occurs gradually in tbp1-1 mutants over four generations (Hwang and Cho, 2007). Mutants for telomerase were also shown to show a loss of telomeric DNA of approximately 500bp per generation along with displaying a discrete banding pattern (Riha et al., 2001). However, it would be expected that knocking out core telomere associated proteins would lead to an immediate and severe effect. This has been shown in many studies where severe telomere defects were observed in $K u 70, \operatorname{stn} 1, c t c 1$, and DNA polymerase $\alpha$ mutants, these are observed within one generation (Riha et al., 2002; Song et al., 2008; Surovtseva et al., 2009; Derboven et al., 2014).

We further examined telomere-end structure as depletion of telomere binding proteins may impair chromosome end protection and integrity of blunt-ended telomeres that are present in plants (Kazda et al., 2012). The current model for chromosome end protection in Arabidopsis suggests that telomeres at the leading end are protected from nucleotytic processing by the $\mathrm{Ku}$ heterodimer immediately after DNA replication. Because of this, lagging end telomeres in plants are thought to generate classical T-loop structures, whereas leading end telomeres remain blunt-ended and protected by Ku. A hairpin ligation assay was previously developed by Kazda et al. (2012) to detect the presence of blunt ends at Arabidopsis telomeres. Briefly, hairpin sequences containing a BamHI site are ligated to blunt-ended telomeres and DNA is digested with AluI to liberate telomeres. Hairpin structures are then subject to alkaline electrophoresis which produces a shift in the higher molecular weight TRF signal. Digestion with BamHI shows that these events are the result of ligation of the hairpin to natural telomeric ends.

Because of the essential role of telomere binding proteins in telomere protection, we reasoned that their inactivation would lead to resection of blunt ended telomeres. However, no observable difference was seen in the presence of blunt ends in tbp1 trfl9 double mutants using blunt end and short-overhang containing hairpins (Figure 2C). These data argue that absence of TBP1 and TRFL9 does not have any discernible effect on telomere structure. 


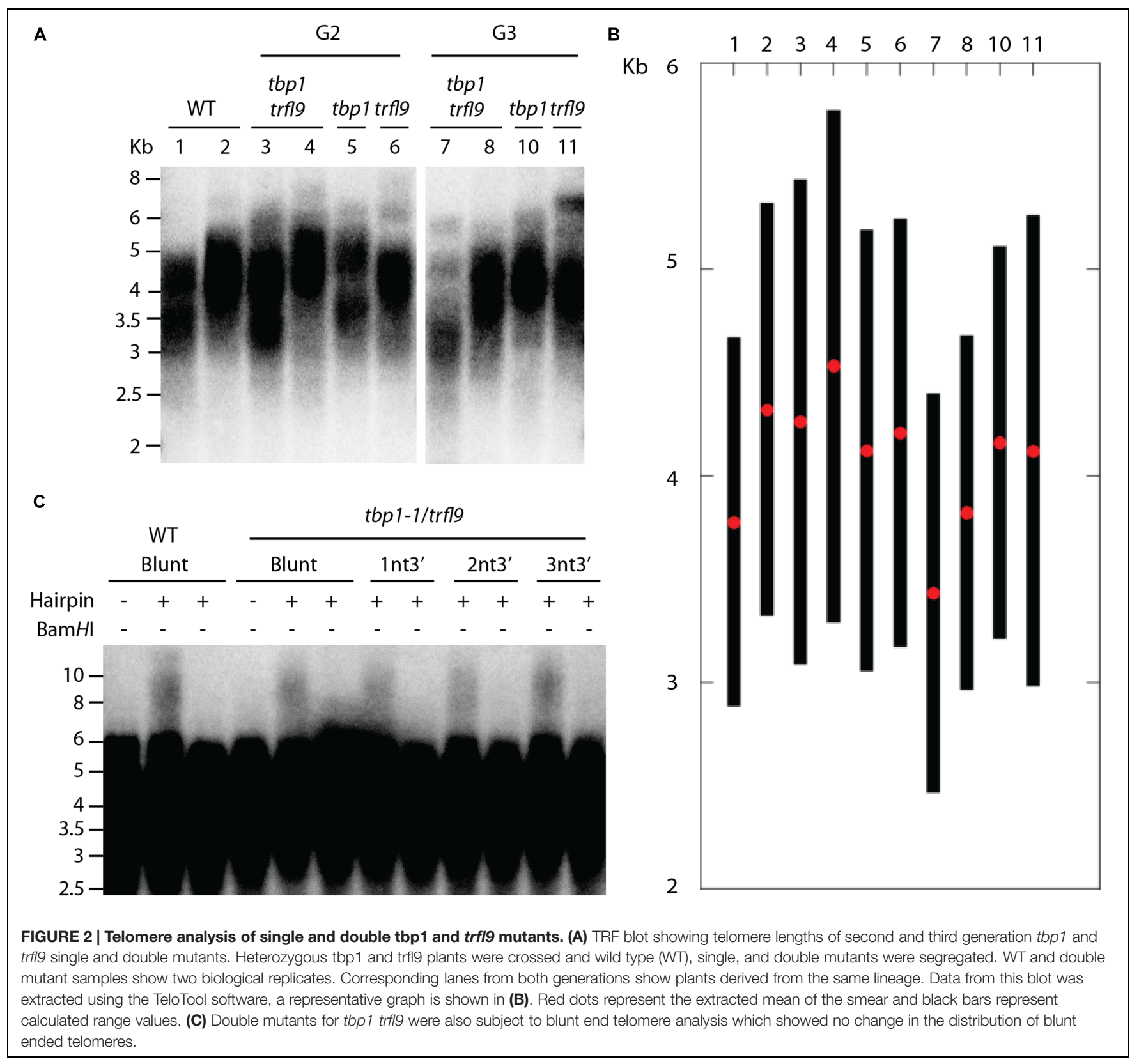

\section{Multiple Combinations of Quadruple, Quintuple, and Sextuple Mutants Showed No Large Effect on Telomere Length}

Because of the sequence similarities between the TRFL proteins, it is possible that other TRFL homologs compensate the functions of TBP1 and TRFL9 in their absence. Therefore, we decided to construct Arabidopsis plants with multiple mutant combinations of the genes in the group I TRFL family. Because generation of sextuple mutants would require extensive screening of a large number of plants in segregating populations, we decided to take advantage of centromere induced genome elimination to produce haploid F2 plants (Ravi and Chan, 2010). Frequency of any quadruple mutant combination among such haploids is $1 / 16$ as opposed to $1 / 256$ in a diploid F2 population.

Centromere induced genome elimination involves generation of haploids by crossing diploid plants as male to the cenh3/GFPtailswap haploid inducer. Single T-DNA insertion mutants were selected for each of the six candidate proteins. In addition to $\operatorname{trfl} 9$ and $t b p 1$ alleles which were already mentioned, $\operatorname{trp} 1$ (SALK_125033), trfl1 (SALK_052864), trfl2 (SAIL_73_G01), and trfl4 (SAIL_73_F07) mutants were also obtained. In order to combine all alleles into the same plant, we first created three combinations of double heterozygous mutants $\left(\operatorname{trp} 1^{++/-}\right.$ $\operatorname{trfl} 1^{+/-}, \operatorname{trfl} 2^{+/-} \operatorname{trfl} 4^{+/-}$, and $\left.\operatorname{tbp} 1^{+/-} \operatorname{trfl9^{+/-}}\right)$. Next, we generated two combinations of quadruple mutants, and finally quintuple and sextuple mutants as illustrated in the 


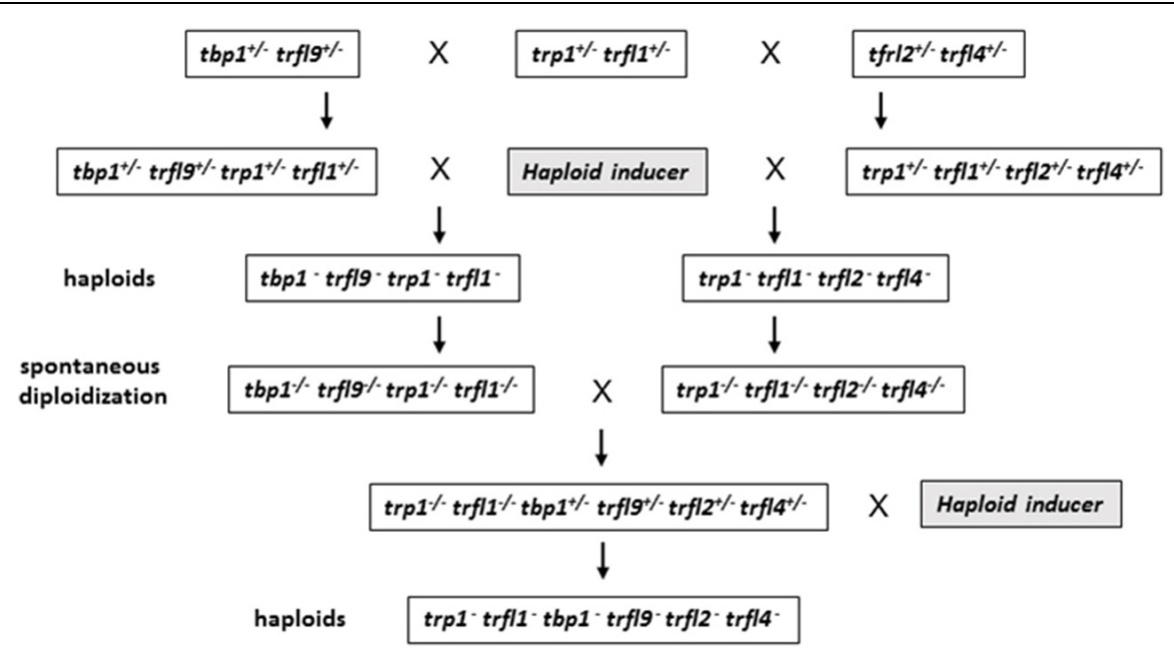

FIGURE 3 | Diagram illustrating crossing scheme for generating multiple TRFL mutants by using centromere mediated genome elimination. See text for details.

crossing scheme (Figure 3). Heterozygous quadruple mutants were then crossed as male to cenh3/GFP-tailswap plants; homozygous quadruple haploids were obtained along with the WT combination. Diploid seeds were obtained from mutant and WT haploids by spontaneous diploidization (Figure 3). First, two single doubled haploid plants were tested by TRF analysis for WT, $\operatorname{tbp} 1^{-/-} \operatorname{trfl9^{-/}-} \operatorname{trp} 1^{-/-} \operatorname{trfl1^{-/-}}$ and $\operatorname{trp} 1^{-/-}$ $\operatorname{trfl} 1^{-/-} \operatorname{trfl} 2^{-/-} \operatorname{trfl} 4^{-/-}$combinations (Second generation without functional protein, Figure 4A). Seeds were collected from these plants and pooled DNA from 5 plants was used for TRF analysis in the following generation (Third generation, Figure 4B). Terminal restriction fragment analysis of resulting tbp $1^{-/-} \operatorname{trfl9^{-/-}} \operatorname{trp} 1^{-/-} \operatorname{trfl1^{-/-}}$ and $\operatorname{trp} 1^{-/-} \operatorname{trfl}^{-/-}$ $\operatorname{trfl} 2^{-/-} \operatorname{trfl} 4^{-/-}$lines showed no effect on telomere length regulation (Figure 4).

Next, we created lines with disruptions in the entire gene family. For this, both quadruple homozygous mutant lines were crossed generating F1 plants that were homozygous for trp1 trfl1 mutations, but segregating for the other four alleles (Figure 3). The haploid induction process was repeated by crossing these plants to the cenh3/GFP-tailswap plants and segregating quintuple and sextuple haploid plants. Individual quintuple and sextuple haploid plants were fully viable and exhibited neither retarded growth in comparison to haploid plants that segregated as WT, nor defects typical for plants with dysfunctional telomeres (Riha et al., 2001; Surovtseva et al., 2009; Derboven et al., 2014). TRF analysis did not reveal drastic changes in telomere length in these mutants (Figure 5), although observed variation seen among individual samples suggests that sextuple mutants could display a higher level of telomere length variation compared to WT. The telomere lengths observed here, however, all lie within the natural telomere length limits seen in Col-0 lines and natural variation amongst diverse Arabidopsis accessions was also shown to vary between approximately 1 and $9 \mathrm{~kb}$ (Fulcher et al., 2015). Normal growth and lack of a clear telomere length deviation in sextuple mutants demonstrates that the Group II TRFL protein family does not play a major role in telomere maintenance in A. thaliana.

\section{Phylogeny of Telobox Containing Proteins in the Plant Kingdom}

Our genetic analysis excluded the possibility that the Group I TRFL protein family harbors functional counterparts of human TRF1/2. Thus, the candidate protein(s) may be encoded by one of the other two telobox families. It is expected that that the bona fide telomere binding protein will be highly conserved in plants. To look at evolution of telobox protein families, we performed systematic phylogenetic analysis of all telobox containing proteins in sequenced genomes representing different phylogenetic groups within plant kingdom. In this analysis we included A. thaliana and Oryza sativa as representatives of dicot and monocot angiosperm plants, respectively, Selaginella moellendorffii representing the oldest branch in the clade of vascular plants, moss Physcomitrella patens and two unicellular green algae, Coccomyxa subellipsoidea and Ostreococcus lucimarinus. Phylogeny based on whole protein alignments revealed presence of the all three telobox protein families already in the moss $P$. patens and separation of TRFL and $\mathrm{Smh} / \mathrm{TRB}$ is apparent already in unicellular algae (Figure 6). This demonstrates ancient origin of the three telobox protein families and their diversification early in evolution of the plant lineage. Hence, telomere function can be associated with either of the remaining two telobox families.

\section{DISCUSSION}

Homologs of TRF1 and TRF2, the double stranded telomere binding proteins central to the shelterin complex, have not been clearly characterized so far in Arabidopsis. These proteins form the core part of shelterin and are essential for telomere 


\section{A}
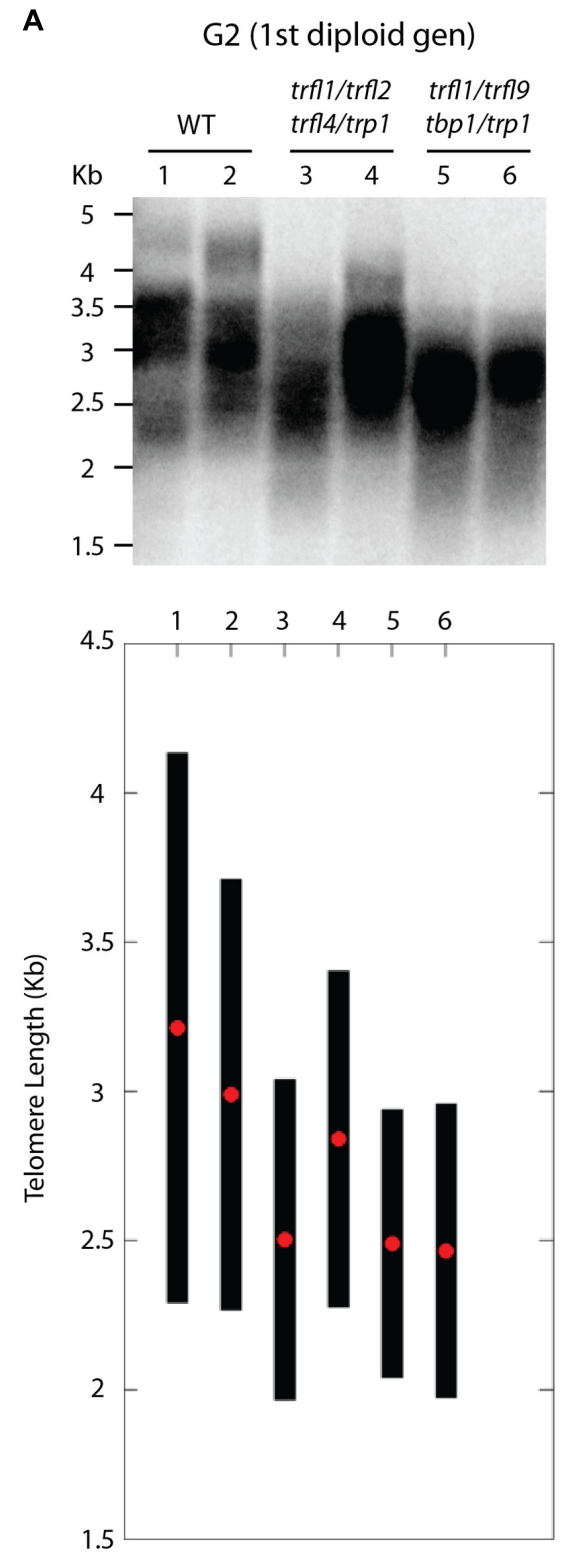

B


FIGURE 4 | Telomere length analysis of quadruple trfl1 trfl2 trfl4 trp1 and trfl1 trfl9 tbp 1 trp1 mutants. TRF blots show telomere lengths from second generation (A) and third generation (B) plants along with WT controls. Blots from the Second generation show TRF profiles of two individual doubled haploid plants. DNA was pooled from 5 plants in the next generation and subject to TRF analysis in the third generation. Corresponding lanes of both blots represent plants of the same lineage. Graphs plotted from TeloTool analysis are shown below respective blots. Red dots represent the extracted mean and black bars show calculated range values.

maintenance and function. Cells expressing dominant negative alleles and conditional knockouts of TRF2 exhibit telomere fusions and telomere length defects demonstrating an essential role of TRF2 in telomere protection (van Steensel et al., 1998; Celli and de Lange, 2005). Functional studies of TRF1 indicate a role of the protein in telomere replication and length regulation (van Steensel and de Lange, 1997; Sfeir et al., 2009) TRFL proteins described in Arabidopsis highlighted a group of potential candidates containing C-terminal telobox motif and plant specific extension domain (Karamysheva et al., 2004). These proteins also bind to telomeric DNA in vitro and the telobox domain is important for this interaction. In addition, studies have shown that disruption of similar proteins in rice, tobacco and tomato leads to telomeric and developmental phenotypes. Transformation of tobacco BY2 cells with 35S:LeTBP1 from tomato was reported to result in telomere shortening from 15-55 kbps to $15-35 \mathrm{kbps}$ (Moriguchi et al., 2006). In a later study, knockdowns of LeTBP1 in tomato showed defects in fruit development and genomic instability, no changes in telomere length were observed in these plants (Moriguchi et al., 


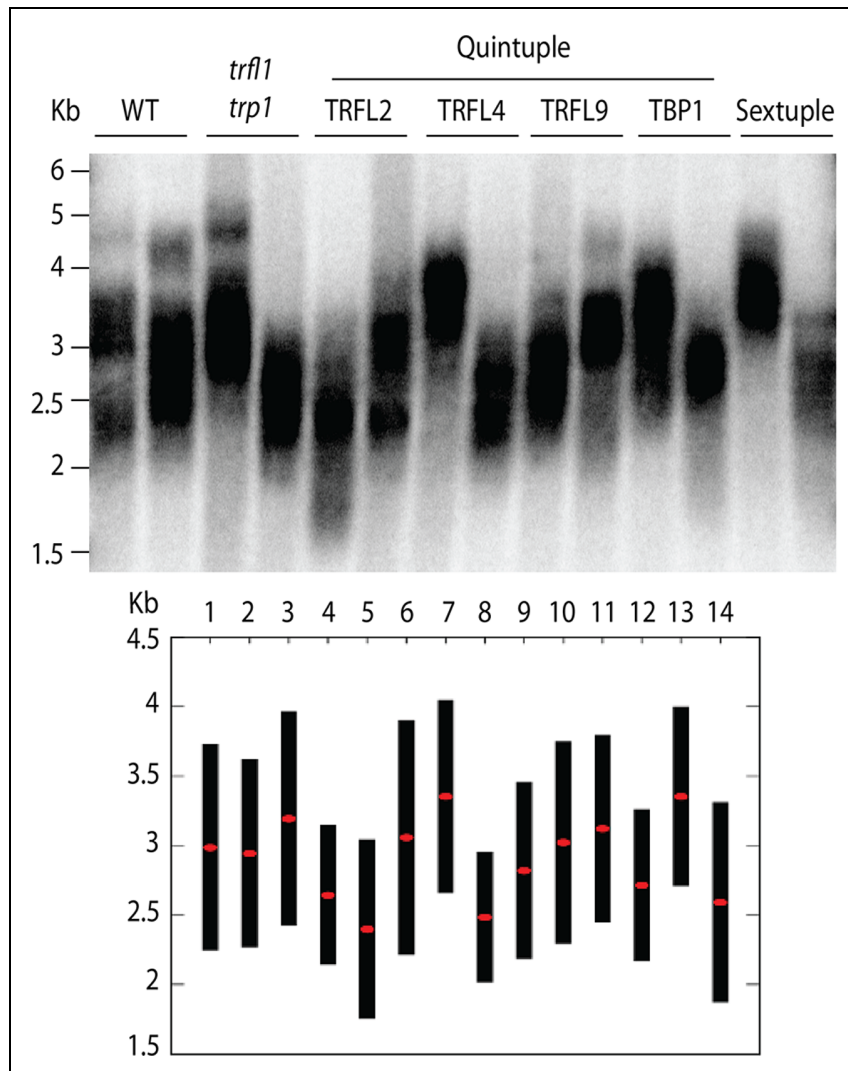

FIGURE 5 | Telomere length analysis of quintuple and sextuple mutants. TRF blot (upper panel) shows telomeric smears from WT, double trfl1 trp1, quintuple (noted gene represents WT locus) and complete sextuple knockouts. DNA was extracted from single segregated haploid plants.

TeloTool analysis is shown (lower panel) where red dots show extrapolated mean and black bars shown range values.

2011). It could be, however, that in these studies, the TRF assay is not sensitive enough to detect small changes that occur in the already long telomeres of tobacco and tomato. Characterization of RICE TELOMERE BINDING PROTEIN1 (RTBP1) showed telomere elongation in first generation RTBP1 knockouts along with anaphase bridges, growth retardation, and floral defects in later generations (Hong et al., 2007). A similar result was reported in Arabidopsis showing knockouts of AtTBP1 undergoing telomere elongation over four generations (Hwang and Cho, 2007). However, the presence of tbp1-1 in the Ws background complicates telomere length analysis as this accession has previously shown to display a bimodal telomere length distribution in WT plants (Shakirov and Shippen, 2004). Because of these previously reported phenotypes of these candidate telomere binding proteins in Arabidopsis and other plant species, in vitro telomeric duplex binding activity, and the high level of sequence conservation, it was expected that the Group I TRFL family comprises the canonical duplex telomere binding proteins.

However, in this study we show that knockouts of all six members of the family in Arabidopsis do not exhibit any obvious changes in telomere length or functionality. Thus, it

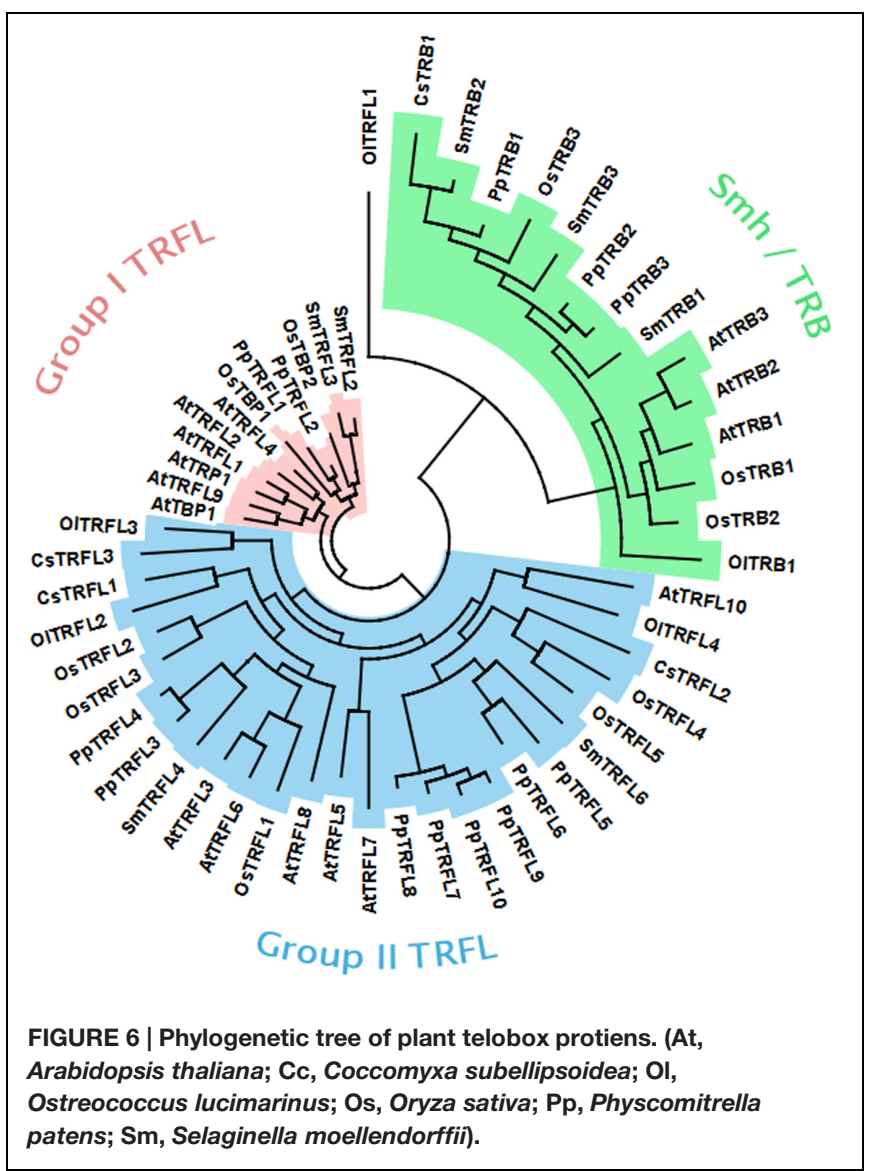

can be concluded that, at least in Arabidopsis, Group I TRFL family does not play a major role in telomere biology. The previously reported in vitro telomere binding of this group suggests there is association with telomeric DNA, although an effect on function has not been observed. Although studies in tobacco, rice, and tomato reported telomere phenotypes associated with knock-outs or overexpressing Group I TRFL proteins (Yang et al., 2004; Moriguchi et al., 2006; Hong et al., 2007), these effects are relatively mild and may reflect only an auxiliary function of these proteins at telomeres. Instead, these proteins may act as transcription factors as promoters of a number of genes are known to contain a short stretch of telomeric sequences (Tremousaygue et al., 1999). Hence, other proteins likely form the core structure of telomeric chromatin in plants.

The question remains as to what proteins comprise the telomere capping complex in Arabidopsis. The Smh/TRB proteins may be the next prime suspects. Phylogenetic analysis shows that these proteins are present in all plant taxonomic units including unicellular green algae suggesting that they may be associated with a fundamental biological function. Three $\mathrm{Smh} / \mathrm{TRB}$ genes with an N-terminal telobox domain have been found in Arabidopsis and have shown to exhibit in vitro binding to telomeric DNA (Schrumpfova et al., 2004; Mozgova et al., 2008; Hofr et al., 2009). Recently, Arabidopsis TRB1 was found to bind to telomeric sequences in vivo through 
immunolocalization studies in tobacco cells (Schrumpfova et al., 2014). One caveat with this approach is that telomeres in tobacco reach far greater lengths than with Arabidopsis $(\sim 5$ and $150 \mathrm{~kb}$ respectively). Association with telomeric DNA may, therefore, not be necessarily for telomere specific functions and can similarly colocalize with non-telomeric sequences. Chromatin Immunoprecipitation (ChIP) studies performed within the same paper, however, confirm binding to telomeric sequences in Arabidopsis. With this evident telomere binding capacity and interaction with Potlb and the N-Terminus of TERT, SMH proteins also show promise as telomere binding components of Arabidopsis telomeres (Kuchar and Fajkus, 2004; Schrumpfova et al., 2014). Telomere length defects are also described for $t r b 1$ mutants although the effect is relatively small after five generations of selfing (Schrumpfova et al., 2014). This could mean redundancy amongst the SMH family of proteins. Additionally, it is possible that members of the tested group 1 TRFL proteins are redundant with SMH/TRB proteins. Functional analysis of other members of this family should clarify the role of these proteins in telomere maintenance.

\section{REFERENCES}

Bilaud, T., Koering, C. E., Binet-Brasselet, E., Ancelin, K., Pollice, A., Gasser, S. M., et al. (1996). The telobox, a Myb-related telomeric DNA binding motif found in proteins from yeast, plants and human. Nucleic Acids Res. 24, 1294-1303. doi: 10.1093/nar/24.7.1294

Celli, G. B., and de Lange, T. (2005). DNA processing is not required for ATMmediated telomere damage response after TRF2 deletion. Nat. Cell Biol. 7, 712-718. doi: $10.1038 /$ ncb1275

de Lange, T. (2005). Shelterin: the protein complex that shapes and safeguards human telomeres. Genes Dev. 19, 2100-2110. doi: 10.1101/gad.1346005

Derboven, E., Ekker, H., Kusenda, B., Bulankova, P., and Riha, K. (2014). Role of STN1 and DNA polymerase alpha in telomere stability and genome-wide replication in Arabidopsis. PLoS Genet. 10:e1004682. doi: 10.1371/journal.pgen.1004682

Fulcher, N., Teubenbacher, A., Kerdaffrec, E., Farlow, A., Nordborg, M., and Riha, K. (2015). Genetic architecture of natural variation of telomere length in Arabidopsis thaliana. Genetics 199, 625-635. doi: 10.1534/genetics.114.172163

Gohring, J., Fulcher, N., Jacak, J., and Riha, K. (2014). TeloTool: a new tool for telomere length measurement from terminal restriction fragment analysis with improved probe intensity correction. Nucleic Acids Res. 42, e21. doi: 10.1093/nar/gkt1315

Hofr, C., Sultesova, P., Zimmermann, M., Mozgova, I., Prochazkova Schrumpfova, P., Wimmerova, M., et al. (2009). Single-Myb-histone proteins from Arabidopsis thaliana: a quantitative study of telomerebinding specificity and kinetics. Biochem. J. 419, 221-228. doi: 10.1042/BJ20 082195

Hong, J. P., Byun, M. Y., Koo, D. H., An, K., Bang, J. W., Chung, I. K., et al. (2007). Suppression of RICE TELOMERE BINDING PROTEIN 1 results in severe and gradual developmental defects accompanied by genome instability in rice. Plant Cell 19, 1770-1781. doi: 10.1105/tpc.107.051953

Hwang, M. G., and Cho, M. H. (2007). Arabidopsis thaliana telomeric DNAbinding protein 1 is required for telomere length homeostasis and its Mybextension domain stabilizes plant telomeric DNA binding. Nucleic Acids Res. 35, 1333-1342. doi: 10.1093/nar/gkm043

Karamysheva, Z. N., Surovtseva, Y. V., Vespa, L., Shakirov, E. V., and Shippen, D. E. (2004). A C-terminal Myb extension domain defines a novel family of double-strand telomeric DNA-binding proteins in Arabidopsis. J. Biol. Chem. 279, 47799-47807. doi: 10.1074/jbc.M407938200

Kazda, A., Zellinger, B., Rossler, M., Derboven, E., Kusenda, B., and Riha, K. (2012). Chromosome end protection by blunt-ended telomeres. Genes Dev. 26, 1703-1713. doi: 10.1101/gad.194944.112

\section{AUTHOR CONTRIBUTIONS}

NF designed and performed the experiments and wrote the paper. KR designed the experiments, performed phylogenetic analysis and wrote the paper.

\section{FUNDING}

This work was supported by the Austrian Science Fund (grant FWF \#Y418-B03), the EMBO Installation Grant (1304130933) and the program SoMoPro II (3SGA5833) co-financed by EU and the South Moravia Region.

\section{SUPPLEMENTARY MATERIAL}

The Supplementary Material for this article can be found online at: http://journal.frontiersin.org/article/10.3389/fgene. 2015.00349

Ko, S., Jun, S. H., Bae, H., Byun, J. S., Han, W., Park, H., et al. (2008). Structure of the DNA-binding domain of NgTRF1 reveals unique features of plant telomerebinding proteins. Nucleic Acids Res. 36, 2739-2755. doi: 10.1093/nar/gkn030

Ko, S., Yu, E. Y., Shin, J., Yoo, H. H., Tanaka, T., Kim, W. T., et al. (2009). Solution structure of the DNA binding domain of rice telomere binding protein RTBP1. Biochemistry 48, 827-838. doi: 10.1021/bi801270g

Kuchar, M., and Fajkus, J. (2004). Interactions of putative telomere-binding proteins in Arabidopsis thaliana: identification of functional TRF2 homolog in plants. FEBS Lett. 578, 311-315. doi: 10.1016/j.febslet.2004.11.021

Marian, C. O., Bordoli, S. J., Goltz, M., Santarella, R. A., Jackson, L. P., Danilevskaya, O., et al. (2003). The maize Single myb histone 1 gene, Smh1, belongs to a novel gene family and encodes a protein that binds telomere DNA repeats in vitro. Plant Physiol. 133, 1336-1350. doi: 10.1104/pp.103.026856

Moriguchi, R., Kanahama, K., and Kanayama, Y. (2006). Characterization and expression analysis of the tomato telomere-binding protein LeTBP1. Plant Sci. 171, 166-174. doi: 10.1016/j.plantsci.2006.03.010

Moriguchi, R., Ohata, K., Kanahama, K., Takahashi, H., Nishiyama, M., and Kanayama, Y. (2011). Suppression of telomere-binding protein gene expression represses seed and fruit development in tomato. J. Plant Physiol. 168, 1927-1933. doi: 10.1016/j.jplph.2011.05.009

Mozgova, I., Schrumpfova, P. P., Hofr, C., and Fajkus, J. (2008). Functional characterization of domains in AtTRB1, a putative telomere-binding protein in Arabidopsis thaliana. Phytochemistry 69, 1814-1819. doi: 10.1016/j.phytochem.2008.04.001

Ravi, M., and Chan, S. W. (2010). Haploid plants produced by centromeremediated genome elimination. Nature 464, 615-618. doi: 10.1038/nature08842

Ravi, M., Marimuthu, M. P., Tan, E. H., Maheshwari, S., Henry, I. M., MarinRodriguez, B., et al. (2014). A haploid genetics toolbox for Arabidopsis thaliana. Nat. Commun. 5, 5334. doi: 10.1038/ncomms6334

Riha, K., Mcknight, T. D., Griffing, L. R., and Shippen, D. E. (2001). Living with genome instability: plant responses to telomere dysfunction. Science 291, 1797-1800. doi: 10.1126/science. 1057110

Riha, K., Watson, J. M., Parkey, J., and Shippen, D. E. (2002). Telomere length deregulation and enhanced sensitivity to genotoxic stress in Arabidopsis mutants deficient in Ku70. EMBO J. 21, 2819-2826. doi: 10.1093/emboj/21.11.2819

Schrumpfova, P., Kuchar, M., Mikova, G., Skrisovska, L., Kubicarova, T., and Fajkus, J. (2004). Characterization of two Arabidopsis thaliana myb-like proteins showing affinity to telomeric DNA sequence. Genome 47, 316-324. doi: 10.1139/g03-136

Schrumpfova, P. P., Vychodilova, I., Dvorackova, M., Majerska, J., Dokladal, L., Schorova, S., et al. (2014). Telomere repeat binding proteins are functional 
components of Arabidopsis telomeres and interact with telomerase. Plant J. 77, 770-781. doi: 10.1111/tpj.12428

Seymour, D. K., Filiault, D. L., Henry, I. M., Monson-Miller, J., Ravi, M., Pang, A., et al. (2012). Rapid creation of Arabidopsis doubled haploid lines for quantitative trait locus mapping. Proc. Natl. Acad. Sci. U.S.A. 109, 4227-4232. doi: 10.1073/pnas.1117277109

Sfeir, A., Kosiyatrakul, S. T., Hockemeyer, D., Macrae, S. L., Karlseder, J., Schildkraut, C. L., et al. (2009). Mammalian telomeres resemble fragile sites and require TRF1 for efficient replication. Cell 138, 90-103. doi: 10.1016/j.cell.2009.06.021

Shakirov, E. V., and Shippen, D. E. (2004). Length regulation and dynamics of individual telomere tracts in wild-type Arabidopsis. Plant Cell 16, 1959-1967. doi: 10.1105/tpc.104.023093

Song, X., Leehy, K., Warrington, R. T., Lamb, J. C., Surovtseva, Y. V., and Shippen, D. E. (2008). STN1 protects chromosome ends in Arabidopsis thaliana. Proc. Natl. Acad. Sci. U.S.A. 105, 19815-19820. doi: 10.1073/pnas.0807867105

Surovtseva, Y. V., Churikov, D., Boltz, K. A., Song, X., Lamb, J. C., Warrington, R., et al. (2009). Conserved telomere maintenance component 1 interacts with STN1 and maintains chromosome ends in higher eukaryotes. Mol. Cell 36, 207-218. doi: 10.1016/j.molcel.2009.09.017

Tremousaygue, D., Manevski, A., Bardet, C., Lescure, N., and Lescure, B. (1999). Plant interstitial telomere motifs participate in the control of gene expression in root meristems. Plant J. 20, 553-561. doi: 10.1046/j.1365-313X.1999.00627.x

van Steensel, B., and de Lange, T. (1997). Control of telomere length by the human telomeric protein TRF1. Nature 385, 740-743. doi: 10.1038/385740a0

van Steensel, B., Smogorzewska, A., and De Lange, T. (1998). TRF2 protects human telomeres from end-to-end fusions. Cell 92, 401-413. doi: 10.1016/S00928674(00)80932-0
Wang, R. C., Smogorzewska, A., and De Lange, T. (2004). Homologous recombination generates T-loop-sized deletions at human telomeres. Cell 119, 355-368. doi: 10.1016/j.cell.2004.10.011

Watson, J. M., and Riha, K. (2010). Comparative biology of telomeres: where plants stand. FEBS Lett. 584, 3752-3759. doi: 10.1016/j.febslet.2010. 06.017

Wijnker, E., Van Dun, K., De Snoo, C. B., Lelivelt, C. L., Keurentjes, J. J., Naharudin, N. S., et al. (2012). Reverse breeding in Arabidopsis thaliana generates homozygous parental lines from a heterozygous plant. Nat. Genet. 44, 467-470. doi: 10.1038/ng.2203

Yang, S. W., Kim, S. K., and Kim, W. T. (2004). Perturbation of NgTRF1 expression induces apoptosis-like cell death in tobacco BY-2 cells and implicates NgTRF1 in the control of telomere length and stability. Plant Cell 16, 3370-3385. doi: 10.1105/tpc.104.026278

Zellinger, B., and Riha, K. (2007). Composition of plant telomeres. Biochim. Biophys. Acta 1769, 399-409. doi: 10.1016/j.bbaexp.2007. 02.001

Conflict of Interest Statement: The authors declare that the research was conducted in the absence of any commercial or financial relationships that could be construed as a potential conflict of interest.

Copyright (c) 2016 Fulcher and Riha. This is an open-access article distributed under the terms of the Creative Commons Attribution License (CC BY). The use, distribution or reproduction in other forums is permitted, provided the original author(s) or licensor are credited and that the original publication in this journal is cited, in accordance with accepted academic practice. No use, distribution or reproduction is permitted which does not comply with these terms. 